\title{
Assessing the benefits of natriuretic peptides-guided therapy in chronic heart failure
}

\author{
Giuseppe Cocco, Paul Jerie \\ Cardiology Office, Rheinfelden, Switzerland
}

\begin{abstract}
Heart failure (HF) is a major public heart burden among the ageing population. Optimizing management of patients remains challenging despite many advances in therapy for this pathology. Natriuretic peptides (NPS) are related to cardiac morbidity and mortality and their use in guiding treatment might help. Most data on the value of NPs-guided therapy in chronic HF came from centers with high experience in the therapy of HF. Ninety percent of patients had preserved left ventricular function. The story is just too complex to have the final answer. The numbers of treated patients is insufficient to allow a final decision. Most data derive from centers with high skills and were obtained with different assays, different protocols. Many questions are open. Can similar results be obtained in less specialized centers? It is undecided which NP should be used and how high should be the levels to guide the therapy. Which patients might especially benefit from this approach? Is the approach useful in patients with reduced systolic function? Is the strategy as useful in the elderly as in younger patients? In spite of these limitations, available data suggest that it is reasonable to consider the use of NPs to guide the therapy of HF with preserved systolic function. In order to answer some of the questions, a multicenter, prospective study began in January 2013. However, NP guided therapy in chronic HF will only find acceptance in clinical practice if its use results in therapeutic consequences. (Cardiol J 2015; 22, 1: 5-11)
\end{abstract}

Key words: natriuretic peptides, chronic heart failure

\section{Introduction}

Chronic heart failure $(\mathrm{CHF})$ is a major public health burden, especially in elderly patients. While modern guidelines on CHF emphasize the importance of achieving maximally tolerated doses of available drugs [1-4], in practice current medical treatment is associated with many limitations and is often suboptimal. Especially the highest risk-patients are often insufficiently treated, a fact called 'risk treatment' mismatch [5]. Suboptimal therapy is due to multiple reasons. In high-risk patients, side-effects of drugs are particularly frequent. In HF, the clinical assessment is often misleading and reliable markers to titrate the therapy are not regularly used. Natriuretic peptides (NPs) are valid diagnostic and prognostic markers in $\mathrm{HF}$ and it has been suggested that measuring NPs concentrations should be useful to guide the therapy [6-14]. Results derived from this strategy have been largely published. We review and discuss the present knowledge.

\section{Natriuretic peptides}

Recently, Volpe et al. [15] have written a clinical update which is largely based on their experience which is proven by 13 personal publications.

Address for correspondence: Giuseppe Cocco, MD, Cardiology Office, Marktgasse 10A, CH-4310 Rheinfelden, Switzerland, tel: +00 416183145 55; fax: +00 416183397 56, e-mail: praxis@cocco.ch 
Table 1. Authors who published the large majority of papers, treated $>8,000$ patients, and are members of Working Groups on Heart Failure.

\begin{tabular}{ll}
\hline References number & \multicolumn{1}{c}{ Frequent authors } \\
\hline$[3,4,5,8,9,18,24,25,26,27,28,29,30$, & Baggish AL, Cleland JG, Gaggin HK, Januzzi JL Jr, McMurray JJ, \\
$31,32,33,34,35,36,37,38,39,40,49]$ & Troughton RW \\
{$[40,41,42]$} & Brunner-La Rocca H-P, Buser P, Dubach P, Erne P, Estlinbaum W, \\
& Maeder M, Pfisterer ME, Rickenbacher P, Suter T, Vuillomenet A \\
\hline
\end{tabular}

Under physiological conditions in humans NPs are produced primarily in the atria where they have the highest concentration and are mostly stored in granules [16]. However, in response to cardiac pathologies with pressure or volume overload, ventricular myocytes re-express fetal genes and release most of the NPs [17]. Released natriuretic propeptide is cleaved into two major molecules, a bioactive B-type natriuretic peptide (BNP) and an inactive $\mathrm{N}$-terminal pro B-type natriuretic peptide (NT-proBNP). Both NPs are used and readily measured with commercially available assays. Some aspects of their assessment deserve consideration: a) methods differ substantially; b) different antibodies are used in various combinations; c) BNP is measured with different calibrators; d) even assays using the same antibodies run on different instruments and the results vary markedly; e) the different half-lives of BNP and NT-proBNP have an effect on the measurement of serial concentrations. While NT-proBNP assays may have a systematic bias at higher concentrations [18], it seems that it may be more useful than BNP to 'guide' medical therapy in HF [19-22].

\section{Strategy NPs-of guided therapy in HF}

In 1999, Murdoch et al. [23] described the usefulness of plasma BNP concentrations in titrate vasodilator therapy in CHF. Since this early publication many papers have reported results of 'NPs-guided' vs. symptoms-guided therapy in CHF. As shown in Table 1 many of the cardiologists who published results on this argument are members of Working Groups on HF and have co-authored modern Guidelines on the Diagnosis and Therapy of $\mathrm{HF}[3,4]$. Most published data, i.e. 19 publications $[8,9,18,24-41]$ are from these cardiologists. The number of treated patients in these centers outweighs that of patients treated elsewhere and the results from the 'specialized' and 'normal' centers are different and sometimes opposed. We try to explain some differences.

\section{Changes in plasma levels of NPs without therapy}

Sequential measurements have shown that plasma NPs-levels vary daily and only few fluctuations are due to measurements' variability [15, $33,35,36]$. Also fluctuations in NPs levels are not associated with changed symptoms, clinical status or edema [10, 11, 14, 15, 25, 33, 35-38].

Most data were obtained with BNP and it seems possible that the use of NT-proBNP, with its longer half-life would provide better results [19-22].

Most fluctuations are related to changing hemodynamics, such as left ventricular (LV) filling pressure, volume, systolic and diastolic function, and also right ventricular filling pressure [7, 15, 41-45] but NPs values cannot be used to estimate LV filling pressure [25] and do not differentiate between systolic and diastolic dysfunction [18, 25].

Concentrations of NPs fall with increasing body mass index [27], increase with age, with declining renal function, are slightly higher in women $[7-9,15,33-36]$, and are affected by the occurrence of atrial fibrillation [39]. Furthermore, cutoff values between normality and pathology differ between acute and CHF [15, 43, 44].

The values of NPs as prognostic markers may be partially related to their correlation with other risk markers, such as LV function, renal dysfunction, atrial fibrillation, and age [15, 33, $35,39]$. Nonetheless, in CHF, a change in BNP values of $>20 \%$ between monthly follow-ups indicates a clinically important change, greater than would be expected from spontaneous variations [7-9, 15, 42, 43]. Indeed, NPs plasma levels offer a risk prediction that is independent from other factors $[38,43]$. As shown in Table 2, persistently elevated or increasing levels of NPs are consistently associated with an increased risk of cardiac morbidity and hospitalization [37, 43]. In stable CHF increased risk is evident from a BNP level of $100 \mathrm{pg} / \mathrm{mL}$, with each further 
Table 2. Natriuretic peptides as a risk factor for cardiovascular events in chronic heart failure.

\begin{tabular}{ll}
\hline Parameters & \multicolumn{1}{c}{ Value $[\mathrm{pg} / \mathrm{mL}]$} \\
\hline B-type natriuretic peptide & $100 \mathrm{pg} / \mathrm{mL}$, with each further $100 \mathrm{pg} / \mathrm{mL}$ increase associated \\
& $\begin{array}{l}\text { with a } 35 \% \text { increase in risk } \\
200 \mathrm{pg} / \mathrm{mL}, \text { rises almost exponentially with increasing levels, } \\
\begin{array}{l}\text { N-terminal pro B-type } \\
\text { natriuretic peptide }\end{array}\end{array}$ \\
\hline
\end{tabular}

$100 \mathrm{pg} / \mathrm{mL}$ increase associated with a $35 \%$ increase in risk [7, 39, 43-47]. For NT-proBNP the risk is evident from $200 \mathrm{pg} / \mathrm{mL}$ and rises almost exponentially with increasing levels, especially above $100 \mathrm{pg} / \mathrm{mL}[33,44,45,48]$. Table 2 shows the values for NPs which are consistently associated with cardiovascular complications in patients with CHF.

\section{Changes in plasma levels of NPs with therapy}

In CHF, NPs levels have been shown to fall in response to loop diuretics, angiotensin converting enzyme inhibitors, angiotensin receptor blockers and mineral receptor antagonists [29-33, 35-49]. The response to the beta-blocker metoprolol is complex, sometimes with a rise of BNP levels in the first 2-3 months followed by a fall [26]. Treatment with the direct renin inhibitor aliskiren was also found to reduce NT-proBNP levels [28] but this fall was not associated with reduced clinical events [50]. Levels of NPs also fall with exercise [51], lifestyle interventions $[52,53]$ and after cardiac resynchronization therapy [54].

One may assume that in CHF a change in NPs levels should be an important independent predictor of clinical outcomes and provide an accurate estimate of risk. A failure of NPs concentrations to fall in response to the therapy might suggest that the risk is not reduced. Therefore, serial monitoring with NPs levels might deliver powerful information about the response to the therapy and remaining risk [6-8, 15, 31-35, 40, 43-48].

\section{Different results of different studies on NPs-guided therapy}

Results of available publications are conflicting. Table 3 shows the value of NPs-guided therapy in CHF. Positive studies derive almost exclusively from those centers which have treated the overwhelming majority of patients and have published the majority of papers on the argument; most of these authors are members of Working Groups on
Table 3. Value of natriuretic peptides-guided therapy in chronic heart failure.

\begin{tabular}{ll}
\hline Useful & Authors [Reference] \\
\hline Yes & Bhardwaj et al. [31] \\
Yes & Januzzi Jr [33] \\
Yes & Maeder et al. [41] \\
Yes & Sanders-van Wijk et al. [42] \\
Yes & Berger et al. [55] \\
Yes & Jourdain et al. [56] \\
No & Persson et al. [45] \\
No & Eulings et al. [57] \\
No & Karlstrom et al. [58] \\
No & Shah et al. [59] \\
\hline
\end{tabular}

HF (Table 1). While 6 studies [31, 33, 41, 42, 55, 56] reported encouraging trends with the NPs-guided therapy in comparison to conventional care, such as reduced events in younger patients or in patients with reduced LV ejection fraction (in these studies a very low NP level was targeted, both in the intervention arm with NP-guided and in arm without NP-guided therapy), other studies [45, 57-59] presented neutral results. Results derive from different approaches. One of the differences among the studies was the patients' selection. Some centers studied outpatients with CHF, while other selected patients at discharge from the hospital where they had been treated because of an acute cardiac failure. Furthermore, target NPs values were chosen empirically. Many studies did not meet their primary outcomes in treated patients because the chosen basal NPs values were too high to reduce cardiac events. For example, the STARBRITE study [59] used a goal BNP-value near $450 \mathrm{pg} / \mathrm{mL}$ : by the end of the trial the BNP-values were quite similar to those at hospital discharge and all observed changes were within the biological variability of BNP. The chosen BNP-value is questionable because the threshold of risk for BNP is more than half of this value $[7,15$, 39]. It is thus not surprising that cardiac adverse effect rates were high in the BNP-guided arm and 
similar to the values of the conventional therapy. Moreover, the time of collection of basal NPs-values was also different among centers. In the PRIMA study [57], 'individualized baseline' NPs-values were collected at the time of hospital discharge after an acute cardiac decompensation: a high event rate of complications occurred and the NPs-guided care was not useful. The time chosen in this study to get baseline NPs values is questionable, because later experience has shown that NPs-values after an acute cardiac decompensation are considerably higher than values used in stable CHF and higher than the goal that can be achieved with titration of therapy [30-36, 40].

In spite of important differences in trial designs, patients' selection, chosen time, duration of collection, type and concentrations of NPs, the centers which treated the majority of patients [30, $32,55-56$ ] concluded that NPs-guided therapy was usually accompanied by numerically and often statistically greater up-titration or addition of $\mathrm{HF}$ therapies. Because of the deficiencies in care that are well documented, in present therapy of $\mathrm{HF}$ [5] by itself this is a desirable effect. Indeed in the PROTECT study [38], both arms received more intensified therapy than in other studies. Again, it must be remembered that most data came from two 'specialized' centers and from authors who have published modern Cardiac Guidelines on Diagnosis and Therapy of HF (Table 1). The quantity of data and number of publications from these centers outweigh those from other centers and it is uncertain if the results from the Working Groups on $\mathrm{HF}$ can be repeated in less experienced centers.

Not astoundingly, depending on the heterogeneity meta-analyses, the published studies give different results. Altogether, available data [19-22] suggest that BNP may be less useful than NT-proBNP to guide therapy in HF. Indeed, in a meta-analysis [29] NT-proBNP-guided therapy reduced death by $28 \%(\mathrm{p}=0.007)$ and HF-related hospitalization by $47 \%(\mathrm{p}=0.003)$, whereas BNP-guided therapy reduced these outcomes by $19 \%(\mathrm{p}=0.37)$. However, BNP was used in few centers and the majority used NT-proBNP. Thus the comparative merits of the two $p$ values cannot be fully accepted.

NPs are useful in the care of acute HF also in the presence of concomitant diseases [25] but this knowledge should not be extrapolated for their use in CHF [39]. Little is known about the validity of using NPs-guided care in patients with $\mathrm{CHF}$ associated with atrial fibrillation, renal dysfunction and other diseases.
Table 4. Value of natriuretic peptides-guided therapy in elderly patients with chronic heart failure.

\begin{tabular}{ll}
\hline Useful & Authors [Reference] \\
\hline No & Savarese et al. [22] \\
No & Lainchbury et al. [29] \\
No & Pfisterer et al. [40] \\
Yes & Motiwala and Januzzi [35] \\
\hline
\end{tabular}

Elderly patients seem to have very high values of NPs and in a meta-analysis [22] most studies have suggested that the benefits of NPs-guided therapy are primarily limited to younger patients. This effect is especially evident in two studies [29, 40]. On the contrary, another study [36] came to the opposite conclusion and showed that the NPs-guided therapy was more effective in the elderly than in the younger patients. The results are summarized in Table 4. Thus, it is yet unclear if age may affect the use of NPs-guided care. Since CHF is the most frequent cause of morbidity and mortality for patients $>65$ years of age and is a major public heart burden among the ageing population, confirming these findings would reduce the utility of NPs-guided care in elderly patients with CHF.

Lastly, HF with preserved systolic function differs from $\mathrm{HF}$ with reduced systolic function. However, $90 \%$ of patients treated with NPs-guided care had a normal ejection function [39]. Present data seem to indicate that NPs-guides care might be less efficacious in patients with reduced ejection function and high NT-proBNP levels [42, 48]. If confirmed, these findings would reduce the utility of NPs-guided care in this type of CHF.

\section{Safety of NPs-guided therapy}

None of the published studies noted an important increase in untoward reactions which might be related to NPs-guided care. However, the number of treated patients was too small to exclude some side-effects and the two 'specialized' centers using this approach do not represent the use in normal conditions. Therefore, we don't know if the use of NPs-guided cardiac care in less specialized centers will be safe.

\section{NPs-guided therapy and quality of life}

In a study [48] on NPs-guided therapy, no difference was detected in comparison with conventional care in improving the quality of life, but in another study [50] NPs-guided therapy was 
superior to conventional care in improving the quality of life. Thus at present we do not know if NPs-guided therapy is useful in improving the quality of life.

\section{NPs-guided therapy and cardiac function}

Important data show that high NPs levels on the long term might indicate a deleterious LV remodeling [8, 15]. A study [37] has shown that NPs-guided care reduces NPs levels and speculates whether the negative ventricular remodeling might be reduced by NPs-guided care. This is a wishful speculation.

\section{Costs of NPs-guided therapy}

Some studies $[41,60,61]$ show that in ambulatory settings, NPs-guided care would reduce the need of repeated hospitalizations and should be cost-effective. However, studies with different patients, trial designs and in different heath care systems are missing and at present it cannot be concluded that NPs-guided care is cheaper than conventional care [39].

\section{Non-response to NPs-guided therapy}

Some patients do not benefit from NPs-guided care. In some of these patients, selected NPs values were very high and in spite of serial measurements and changes in the medical therapy the cardiovascular complications were insufficiently reduced. It might be thought that 'some' reduction of NPs levels might still be useful. Indeed, in two studies with overall neutral results $[56,57]$ those patients in whom NT-proBNP decreased to a significant extent had a better clinical outcome.

\section{Conclusions, questions, and future}

Optimizing management of patients with CHF remains challenging despite many significant advances in drug and device therapy for this pathology. Although a large body of evidence from clinical trials supports multiple therapies, in 'real-world', utilization of these well-established treatments remains inconsistent and outcomes are suboptimal in patients with CHF. NPs-guided care has been used with the aim to improve insufficient therapy. The story is just too complex to have the final answer. At present, we have data from about 8,000 patients who were treated with this system, and $90 \%$ of them preserved systolic function [39]. The number is insufficient to allow a final decision,
Table 5. Open questions about the natriuretic peptides (NPs)-guided therapy in chronic heart failure (HF).

Should B-type natriuretic peptide or N-terminal
pro B-type natriuretic peptide be used?
How high should be the target NPs value at
selection and during guided therapy?
Is NPs-guided care as useful in the elderly as in
younger patients?
NPs-guided care was mainly used in experienced
centers. Can the strategy be useful in less
specialized centers?
Might patients discharged from the hospital after
a worsening of HF and who are at high risk benefit
from NPs-guided care?
Might high risk patients, such as those with atrial
fibrillation and renal dysfunction, benefit from
NPs-guided care?
NPs-guided care was used mostly in patients with
diastolic HF. Might the approach also be useful in
patients with reduced systolic function?

especially if one considers that most of the data derived from two centers with high skills in the therapy of $\mathrm{HF}$ and that data were obtained with different NPs assays, from different patients, with different sampling protocols, with different follow-up lengths and many more. Several problems exist and must be taken into account. In HF, NPs are produced in the heart and, to a lesser extent, in other organs [15]. The diagnosis of HF encompasses many pathologies and types of disease, and many factors influence the production and amount of NPs. It has been demonstrated that the values of NPs change according to the time of assessment and changing severity of HF [15]. Present data have been collected by using either BNP or NT-proBNP. However, new biomarkers have been introduced, such as galectin-3 and soluble ST2. It has been suggested [39] that these new markers could be useful for the selection of patients who would be likely to benefit from special therapies. In the future we may perhaps use special biomarkers, learn more about the pathophysiology of some cardiac diseases, and thus use a more appropriate therapy.

Still, many questions are open about the NPs-guided therapy for CHF and they are illustrated in Table 5. Because of the unanswered questions, present Cardiology Guidelines from Europe and Australia have not yet given any recommendation on the use of NPs-values to guide therapy in CHF. Many authors who have published the majority of publications on this strategy have also contributed to the Cardiology Guidelines from the American 
College of Cardiology and American Heart Association, and these guidelines give a class IIa recommendation (reasonable to consider) to the NPs-guided care in $\mathrm{CHF}$ and also state that the utility is not established to reduce hospitalization and mortality, a class IIb recommendation [3]. Because of the present uncertainties, the United States National Heart Lung Blood Institute has funded the GUIDing Evidence using Biomarker Intensified Treatment (GUIDE-IT, NCT01685840), a prospective, multicenter, randomized trial that will follow 1,100 patients with $\mathrm{HF}$ and LV ejection fraction $<40 \%$. Patients will be enrolled at the time of discharge from the hospital and will be randomized to either usual care or to NT-proBNP-guided care, with a target value $<1,000 \mathrm{pg} / \mathrm{mL}$. Forty centers from Canada and United States have been participating and the study began in January 2013. Primary endpoints are time to cardiovascular death or recurring hospitalization because of HF. Secondary endpoints are: a) all-cause mortality, b) cumulative morbidity, c) health-related quality of life, and d) costs and cost-effectiveness, and safety.

To conclude, according to the message from experienced authors [39], present data suggest that the use of NPs may be safe and effective. Nonetheless, in spite of many published data, the utility of NPs-guided care in CHF is still unknown. We need answers to the important open questions. NPs-guided therapy of CHF will only find acceptance in clinical practice if its use results in therapeutic consequences, in improvements of prognosis by pharmacologic management.

\section{Acknowledgement}

The authors thank Mrs. Jaqueline Bugmann for the secretarial help.

\section{Conflict of interest: None declared}

\section{References}

1. Lindenfeld J, Albert NM, Boehmer JP et al. HFSA 2010 Comprehensive Heart Failure Practice Guideline. J Card Fail, 2010; 16: e1-e194.

2. Krum H, Jelinek MV, Stewart S, Sindone A, Atherton JJ. 2011 Update to National Heart Foundation of Australia and Cardiac Society of $\mathrm{Au}-$ stralia and New Zealand Guidelines for the prevention, detection and management of chronic heart failure in Australia, 2006. Med J Aust, 2011; 194: 405-409.

3. McMurray JJ, Adamopoulos S, Anker SD et al. ESC Guidelines for the diagnosis and treatment of acute and chronic heart failure 2012: The Task Force for the Diagnosis and Treatment of Acute and Chronic Heart Failure 2012 of the European Society of Cardiology. Developed in collaboration with the Heart Failure Association (HFA) of the ESC. Eur Heart J, 2012; 33: 1787-1847.

4. Yancy CW, Jessup M, Bozkurt B et al. ACCF/AHA Guideline for the Management of Heart Failure: A Report of the American College of
Cardiology Foundation/American Heart Association Task Force on Practice Guidelines. J Am Coll Cardiol, 2013; 62: e147-e239.

5. Lenzen MJ, Boersma E, Reimer WJ et al. Under-utilization of evidence-based drug treatment in patients with heart failure is only partially explained by dissimilarity to patients enrolled in landmark trials: A report from the Euro Heart Survey on Heart Failure. Eur Heart J, 2005; 26: 2706-2713.

6. Bettencourt P, Azevedo A, Pimenta J et al. A. N-terminal-pro-brain natriuretic peptide predicts outcome after hospital discharge in heart failure patients. Circulation, 2004; 110: 2168-2174.

7. Wang TJ, Larson MG, Levy D et al. Plasma natriuretic peptide levels and the risk of cardiovascular events and death. N Engl J Med, 2004; 350: 655-663.

8. Kubanek M, Goode KM, Lanska V et al. The prognostic value of repeated measurement of $\mathrm{N}$-terminal pro-B-type natriuretic peptide in patients with chronic heart failure due to left ventricular systolic dysfunction. Eur J Heart Fail, 2009; 11: 367-377.

9. Cleland JG, McMurray JJ, Kjekshus J et al. Plasma concentration of amino-terminal pro-brain natriuretic peptide in chronic heart failure: Prediction of cardiovascular events and interaction with the effects of rosuvastatin: A report from CORONA (Controlled Rosuvastatin Multinational Trial in Heart Failure). J Am Coll Cardiol, 2009; 54: 1850-1859.

10. Gassanov N, Biesenbach E, Caglayan E et al. Natriuretic peptides in therapy for decompensated heart failure. Eur J Clin Pharmacol, 2012; 68: 223-230.

11. Thygesen K, Mair J, Mueller C et al. Recommendations for the use of natriuretic peptides in acute cardiac care. A position statement from the Study Group on Biomarkers in Cardiology of the ESC Working Group on Acute Cardiac Care. Eur Heart J 2012; 33: 2001-2006.

12. Pruett AE, Lee AK, Patterson JH et al. Biomarker guided therapy for heart failure: Focus on natriuretic peptides. Curr Cardiol Rev, 2015; 11: 80-89.

13. Maisel A, Barnard D, Jaski B et al. Primary results of the HABIT Trial (heart failure assessment with BNP in the home). J Am Coll Cardiol, 2013; 61: 1726-1735.

14. van Veldhuisen DJ, Linssen GC, Jaarsma T et al. B-type natriuretic peptide and prognosis in heart failure patients with preserved and reduced ejection fraction. J Am Coll Cardiol, 2013; 61: 1498-1506.

15. Volpe M, Rubattu S, Burnett J Jr. Natriuretic peptides in cardiovascular diseases: Current use and perspectives. Eur Heart J, 2014; 35 : 419-425.

16. Yasue H, Yoshimura M, Sumida $\mathrm{H}$ et al. Localization and mechanism of secretion of B-type natriuretic peptide in comparison with those of A-type natriuretic peptide in normal subjects and patients with heart failure. Circulation, 1994; 90: 195-203.

17. Hystad ME, Geiran OR, Attramadal H et al. Regional cardiac expression and concentration of natriuretic peptides in patients with severe chronic heart failure. Acta Physiol Scand, 2001; 171: 395-403.

18. O’Neill JO, Bott-Silverman CE et al. B-type natriuretic peptide levels are not a surrogate marker for invasive hemodynamics during management of patients with severe heart failure. Am Heart J, 2005; 149: 363-369.

19. Masson S, Latini R, Anand IS, Vago T et al. Direct comparison of Btype natriuretic peptide (BNP) and amino-terminal proBNP in a large population of patients with chronic and symptomatic heart failure: The Valsartan Heart Failure (Val-HeFT) data. Clin Chem, 2006; 52: 1528-1538.

20. Felker GM, Hasselblad V, Hernandez AF, O'Connor CM. Biomarker-guided therapy in chronic heart failure: A meta-analysis of randomized controlled trials. Am Heart J, 2009; 158: 422-430.

21. Porapakkham P, Zimmet H, Billah B, Krum H. B-type natriuretic peptide-guided heart failure therapy: A meta-analysis. Arch Intern Med, 2010; 170: 507-514.

22. Savarese G, Trimarco B, Dellegrottaglie S et al. Natriuretic peptideguided therapy in chronic heart failure: A meta-analysis of 2,686 patients in 12 randomized trials. PLoS One, 2013; 8: e58287.

23. Murdoch DR, McDonagh TA, Byrne J et al. Titration of vasodilator therapy in chronic heart failure according to plasma brain natriuretic 
peptide concentration: Randomized comparison of the hemodynamic and neuroendocrine effects of tailored versus empirical therapy. Am Heart J, 1999; 138: 1126-1132.

24. Troughton RW, Frampton CM, Yandle TG et al. Treatment of heart failure guided by plasma aminoterminal brain natriuretic peptide (NT-BNP) concentrations. Lancet, 2000; 355: 1126-1130.

25. Chen AA, Wood MJ, Krauser DG et al. NT-proBNP levels, echocardiographic findings, and outcomes in breathless patients: results from the ProBNP Investigation of Dyspnoea in the Emergency Department (PRIDE) echocardiographic substudy. Eur Heart J, 2006; 27: 839-845.

26. Davis ME, Richards AM, Nicholls MG et al. Introduction of metoprolol increases plasma B-type cardiac natriuretic peptides in mild, stable heart failure. Circulation, 2006; 113: 977-985.

27. Bayes-Genis A, Lloyd-Jones DM, van Kimmenade RR et al. Effect of body mass index on diagnostic and prognostic usefulness of aminoterminal pro-brain natriuretic peptide in patients with acute dyspnea. Arch Intern Med, 2007; 167: 400-407.

28. McMurray JJ, Pitt B, Latini R, Maggioni AP et al. Effects of the oral direct renin inhibitor aliskiren in patients with symptomatic heart failure. Circ Heart Fail, 2008; 1: 17-24.

29. Lainchbury JG, Troughton RW, Strangman KM et al. N-terminal proB-type natriuretic peptide-guided treatment for chronic heart failure: Results from the BATTLESCARRED (NT-proBNP-Assisted Treatment to Lessen Serial Cardiac Readmissions and Death) trial. J Am Coll Cardiol, 2009; 55: 53-60.

30. Bhardwaj A, Januzzi JL. Natriuretic peptide-guided management of acutely destabilized heart failure. Rationale and treatment algorithm. Crit Pathways Cardiol, 2009; 8: 146-150.

31. Bhardwaj A, Rehman SU, Mohammed A et al. Design and methods of the Pro-B Type Natriuretic Peptide Outpatient Tailored Chronic Heart Failure Therapy (PROTECT) Study. Am Heart J, 2010; 159: 532-538, e531.

32. Januzzi JL Jr, Rehman S, Mohammed AA et al. Use of amino-terminal pro-B type natriuretic peptide to guide outpatient therapy of patients with chronic left ventricular systolic dysfunction. J Am Coll Cardiol, 2011; 58: 1881-1889.

33. Januzzi JL Jr. Use of biomarkers to 'guide' care in chronic heart failure: What have we learned (so far)? J Card Fail, 2011; 17: 622-625.

34. Troughton RW, Brunner-La Rocca HP et al. B-type natriuretic peptide guided treatment of heart failure and all cause mortality: An individual patient data meta-analysis. Eur Heart J, 2011; 32 (suppl. 1): 313-631.

35. Motiwala SR, Januzzi JL Jr. The role of natriuretic peptides as biomarkers for guiding the management of chronic heart failure. Clin Pharmacol Ther, 2012; 93: 57-67.

36. Gaggin HK, Mohammed AA, Bhardwaj A et al. Heart failure outcomes and benefits of NT-proBNP-guided management in the elderly: Results from the Prospective, Randomized ProBNP Outpatient Tailored Chronic Heart Failure Therapy (PROTECT) Study. J Card Fail, 2012; 18: 626-634.

37. Gaggin HK, Truong QA, Rehman SU et al. Characterization and prediction of natriuretic peptide 'nonresponse' during heart failure management: results from the proBNP Outpatient Tailored Chronic Heart Failure (PROTECT) and the NT-proBNP-Assisted Treatment to Lessen Serial Cardiac Readmissions and Death (BATTLESCARRED) study. Congest Heart Fail, 2013; 19: 135-142.

38. Weiner RB, Baggish AL, Chen-Tournoux A et al. Improvement in structural and functional echocardiographic parameters during chronic heart failure therapy guided by natriuretic peptides: Mechanistic insights from the ProBNP Outpatient Tailored Chronic Heart Failure (PROTECT) study. Eur J Heart Fail, 2013; 15: 342-351.

39. Troughton R, Felker GM, Januzzi JL Jr. Natriuretic peptide-guided heart failure management. Eur Heart J, 2014; 35: 16-24.

40. Pfisterer M, Buser P, Rickli H et al. BNP-guided vs symptom-guided heart failure therapy: the Trial of Intensified vs Standard Medical Therapy in Elderly Patients With Congestive Heart Failure (TIME-CHF) randomized trial. JAMA, 2009; 301: 383-392.

41. Maeder MT, Rickenbacher P, Rickli $\mathrm{H}$ et al. N-terminal pro brain natriuretic peptide-guided management in patients with heart failure and preserved ejection fraction: Findings from the Trial of Intensified versus standard Medical therapy in Elderly patients with Congestive Heart Failure (TIME-CHF). Eur J Heart Fail, 2013; 15: 1148-1156.
42. Sanders-van Wijk S, Muzzarelli S, Neuhaus M et al. Safety and tolerability of intensified, $\mathrm{N}$-terminal pro brain natriuretic peptide-guided compared with standard medical therapy in elderly patients with congestive heart failure: Results from TIME-CHF. Eur J Heart Fail, 2013; 15: 910-918.

43. Araujo JP, Azevedo A, Lourenco P et al. Intraindividual variation of amino-terminal pro-B-type natriuretic peptide levels in patients with stable heart failure. Am J Cardiol, 2006; 98: 1248-1250.

44. Braunwald E. Biomarkers in heart failure. N Engl J Med, 2008; 358: 2148-2159.

45. Persson H, Erntell H, Eriksson B et al. Improved pharmacological therapy of chronic heart failure in primary care: A randomized Study of NT-proBNP Guided Management of Heart Failure-SIGNAL-HF (Swedish Intervention study-Guidelines and NT-proBNP AnaLysis in Heart Failure). Eur J Heart Fail, 2010; 12: 1300-1308.

46. Doust JA, Pietrzak E, Dobson A, Glasziou P. How well does B-type natriuretic peptide predict death and cardiac events in patients with heart failure: Systematic review. BMJ, (Clinical Research) 2005; 330: 625.

47. Latini R, Masson S, Wong M et al. Incremental prognostic value of changes in B-type natriuretic peptide in heart failure. Am J Med, 2006; 119: 70e.23-70e.30.

48. Schou M, Gustafsson F, Kjaer A, Hildebrandt PR. Long-term clinical variation of NT-proBNP in stable chronic heart failure patients. Eur Heart J, 2007; 28: 177-182.

49. Anand IS, Rector TS, Cleland JG et al. Prognostic value of baseline plasma amino-terminal pro-brain natriuretic peptide and its interactions with irbesartan treatment effects in patients with heart failure and preserved ejection fraction: Findings from the I-PRESERVE trial. Circ Heart Fail, 2011; 4: 569-577.

50. Gheorghiade M, Bohm M, Greene SJ et al. Effect of aliskiren on postdischarge mortality and heart failure readmissions among patients hospitalized for heart failure: the ASTRONAUT randomized trial. JAMA 2013;309:1125-1135.

51. Passino C, Severino S, Poletti R et al. Aerobic training decreases B-type natriuretic peptide expression and adrenergic activation in patients with heart failure. J Am Coll Cardiol, 2006; 47: 1835-1839.

52. Cocco G; Pandolfi S, Rousson V. A sufficient weight reduction decreases the cardiovascular complications in diabetic patients with metabolic syndrome. a randomized study of orlistat as an adjunct to lifestyle changes (diet and exercise). Heart Drug, 2005; 5: 68-74.

53. Cocco G, Chu D. Weight reduction reduces NT-proBNP levels in patients with chronic diastolic heart failure. Arch Med Sci, 2007; 2: 112-116.

54. Shanmugam N, Campos AG, Prada-Delgado O et al. Effect of atrioventricular optimization on circulating $\mathrm{N}$-terminal pro brain natriuretic peptide following cardiac resynchronization therapy. Eur J Heart Fail, 2013; 15: 534-542.

55. Berger R, Moertl D, Peter S et al. N-terminal pro-B-type natriuretic peptide-guided, intensive patient management in addition to multidisciplinary care in chronic heart failure a 3-arm, prospective, randomized pilot study. J Am Coll Cardiol, 2010; 55: 645-653.

56. Jourdain, P, Jondeau G, Funck F et al. Plasma brain natriuretic peptide-guided therapy to improve outcome in heart failure. The STARS-BNP multicenter study. J. Am Coll Cardiol, 2007; 49: 1733-1739.

57. Eurlings LW, van Pol PE, Kok WE et al. Management of chronic heart failure guided by individual N-terminal pro-B-type natriuretic peptide targets: results of the PRIMA (Can PRo-brain-natriuretic peptide guided therapy of chronic heart failure IMprove heart fAilure morbidity and mortality?) study. J Am Coll Cardiol, 2010; 56: 2090-2100.

58. Karlstrom P, Alehagen U, Boman K, Dahlstrom U. Brain natriuretic peptide-guided treatment does not improve morbidity and mortality in extensively treated patients with chronic heart failure: Responders to treatment have a significantly better outcome. Eur J Heart Fail, 2011; 13: 1096-1103.

59. Shah MR, Califf RM, Nohria A et al. The STARBRITE trial: A randomized, pilot study of B-type natriuretic peptide-guided therapy in patients with advanced heart failure. J Card Fail, 2011; 17: 613-621.

60. Adlbrecht C, Huelsmann M, Berger R et al. Cost analysis and cost-effectiveness of NT-proBNP-guided heart failure specialist care in addition to home-based nurse care. Eur J Clin Invest, 2011; 41: 315-322.

61. Moertl D, Steiner S, Coyle D, Berger R. Cost-utility analysis of NT-proBNP-guided multidisciplinary care in chronic heart failure. Int J Technol Assess Health Care, 2013; 29: 3-11. 